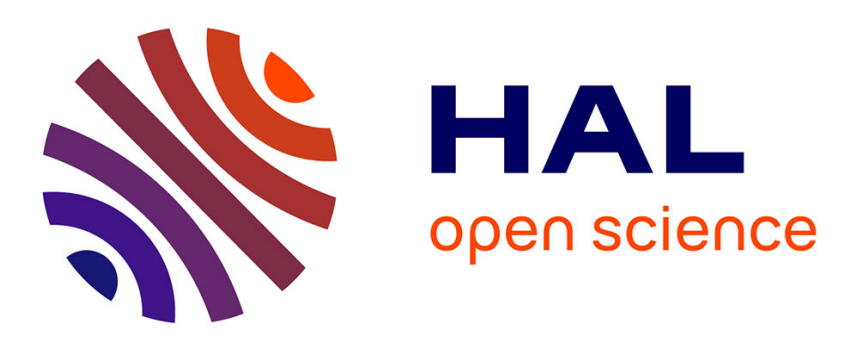

\title{
Construction des lois de fatigue à partir de modèles de forces cohésives : cas de fissures en mode I
}

\author{
Radhi Abdelmoula, Jean-Jacques Marigo, Thibaut Weller
}

\section{To cite this version:}

Radhi Abdelmoula, Jean-Jacques Marigo, Thibaut Weller. Construction des lois de fatigue à partir de modèles de forces cohésives: cas de fissures en mode I. Comptes Rendus Mécanique, 2009, 337, pp.166-172. 10.1016/j.crme.2009.04.002 . hal-00683011

\section{HAL Id: hal-00683011 \\ https://hal.science/hal-00683011}

Submitted on 1 Dec 2021

HAL is a multi-disciplinary open access archive for the deposit and dissemination of scientific research documents, whether they are published or not. The documents may come from teaching and research institutions in France or abroad, or from public or private research centers.
L'archive ouverte pluridisciplinaire HAL, est destinée au dépôt et à la diffusion de documents scientifiques de niveau recherche, publiés ou non, émanant des établissements d'enseignement et de recherche français ou étrangers, des laboratoires publics ou privés.

\section{(ㄷ)(1) $\$$}

Distributed under a Creative Commons Attribution - NonCommerciall 4.0 International 


\title{
Construction des lois de fatigue à partir de modèles de forces cohésives : cas de fissures en mode I
}

\author{
Radhi Abdelmoula ${ }^{a}$, Jean-Jacques Marigo ${ }^{\text {b,* }}$, Thibaut Weller ${ }^{c}$ \\ ${ }^{a}$ LPMTM (UPR-CNRS 9001), Université Paris XIII, avenue Jean-Baptiste-Clément, 93430 Villetaneuse, France \\ b Institut Jean-le-Rond-d'Alembert (UMR 7190), Université Paris VI, 4, place Jussieu, 75005 Paris, France \\ ${ }^{\mathrm{c}}$ LMGC (UMR 5508), Université Montpellier II, CC 048, place Eugène-Bataillon, 34095 Montpellier cedex 5, France
}

\section{Résumé}

Nous reprenons la méthode de construction de lois de fatigue à partir de modèles de forces cohésives et nous l'étendons au cas d'une fissure soumise à un chargement cyclique de mode I. En prenant pour variable mémoratrice l'ouverture cumulée et pour densité d'énergie de surface celle associée au modèle de Dugdale, nous construisons explicitement la loi de fatigue reliant le taux d'avancée de fissure par cycle $\mathrm{d} \ell / \mathrm{d} N$ au facteur d'intensité des contraintes $K_{I}$. En particulier on retrouve une loi de Paris avec un exposant 4 , i.e. d $\ell / \mathrm{d} N=C K_{I}^{4}$, pour des faibles valeurs de $K_{I}$. On montre enfin que ce résultat se généralise à toute une famille d'énergies de surface de type Barenblatt, seule la constante $C$ variant d'un modèle à l'autre.

\section{Abstract}

Construction of fatigue laws from cohesive forces models: the mode I case. We extend the construction of fatigue laws from cohesive forces models to the case of a crack submitted to a mode I cyclic loading. Taking the cumulated opening as the memory variable and the surface energy density associated with Dugdale's model, we explicitly construct the fatigue law which gives the crack growth rate by cycle $\mathrm{d} \ell / \mathrm{d} N$ in terms of the stress intensity factor $K_{I}$. In particular, we recover a Paris law with an exponent 4 , i.e. $\mathrm{d} \ell / \mathrm{d} N=C K_{I}^{4}$, when $K_{I}$ is small. Finally, we show that this result can be generalized to a family of surface energies of Barenblatt's type, the constant $C$ only changes from one model to another.

Mots-clés : Fatigue; Mécanique de la rupture; Approche variationnelle ; Analyse complexe

Keywords: Fatigue; Fracture mechanics; Variational approach; Complex analysis

\footnotetext{
* Auteur correspondant.

Adresses e-mail: radhi@galilee.univ-paris13.fr (R. Abdelmoula), marigo@1mm.jussieu.fr (J.-J. Marigo), weller@1mgc.univ-montp2.fr (T. Weller).
} 


\section{Abridged English version}

We consider a 2D elastic body submitted to a plane strain loading in which a crack propagates along a predefined crack path $\hat{\Gamma}$. The growth of the crack is governed by a cohesive force model of Dugdale-type in which we introduce the so-called cumulated opening $\delta$ as an internal variable memorizing the history of the displacement jump $\llbracket u \rrbracket$ through $\hat{\Gamma}, \dot{\delta}=\llbracket \dot{u} \rrbracket^{+}$. The cohesive normal stress $\sigma \mathbf{n} \cdot \mathbf{n}$ on $\hat{\Gamma}$ is obtained from the Dugdale surface energy density $\phi_{D}(\delta)=$ $\min \left\{\sigma_{c} \delta, G_{c}\right\}$, where $\sigma_{c}$ is the critical stress, $G_{c}$ is the critical energy release rate and the ratio $\delta_{c}:=G_{c} / \sigma_{c}$ is a material characteristic length. Specifically, $\sigma \mathbf{n} \cdot \mathbf{n}=0$ on the part of $\hat{\Gamma}$ where $\delta>\delta_{c}$ whereas $\sigma \mathbf{n} \cdot \mathbf{n}$ is given by (1) on the part of $\hat{\Gamma}$ where $\delta<\delta_{c}$. We assume that $\hat{\Gamma}$, identified with the segment $[0, L]$, can be divided at each time $t$ into three zones: the non-cohesive zone $\Gamma_{t}^{d}$, the cohesive zone $\Gamma_{t}^{c}$ and the sound zone $\Gamma_{t}^{b}$. The cumulated opening is equal to $\delta_{c}$ at the tip of the non-cohesive zone and there is no singularity at the tip of the cohesive zone. When the ratio $\delta_{c} / L$ is small, the problem can be investigated with a two-scale method. Indeed, in the case of a cyclic loading, the crack evolution enjoys the following properties: (i) at each cycle, the growth of the length of the non-cohesive zone is small; (ii) the length of the cohesive zone is always small; (iii) a quasi-stationary regime takes place in the sense that the evolution is approximately the same from a cycle to the next one. Therefore, a great number $N$ of cycles is needed so that the non-cohesive crack length be equal to $\ell$, with $\ell$ of the order of $L$. If we consider cycles close to $N$ and denote by $i$ the $i$ th cycle numbered from $N, i \in \mathbb{Z}$, the corresponding displacement field $\mathbf{u}^{i}$ is in a first approximation independent of $i$, say $\mathbf{u}$. This approximation $\mathbf{u}$ is given by (3) and corresponds to the response of the body on a large scale, i.e. with a non-cohesive crack of length $\ell$ and without any cohesive zone. This field is singular at $\ell$, with a stress intensity factor $K_{I}$ depending on $\ell$ but not on $i$, see (4). But $\mathbf{u}$ is a good approximation of $\mathbf{u}^{i}$ only far from the crack tip. In the same manner, the growth rate of the crack length and the length of the cohesive zone are both small and independent of $i$ at each cycle $i$. They are denoted $\dot{\ell}$ and $d$ respectively. The rate $\dot{\ell}$ can be identified with the macroscopic rate $\mathrm{d} \ell / \mathrm{d} N$. The determination of $\dot{\ell}$ and $d$ requires to obtain a more accurate approximation of $\mathbf{u}^{i}$ near the crack tip. After a rescaling of the zone near the crack tip, the small scale problem giving the approximation $\mathbf{v}^{i}$ of $\mathbf{u}^{i}$ is set on an infinite domain with a semi-infinite straight crack along the axis $y=0$ where $(x, y)$ denotes the new coordinates system. By virtue of the quasi-stationarity property, the $\mathbf{v}^{i}$ 's are related to $\mathbf{v}^{0}$ by (5) and $\mathbf{v}^{0}$ itself must satisfy (6)-(8). Moreover $\mathbf{v}^{0}$ must be non-singular at $x=0$. This problem can be solved in a closed form with the help of the complex potentials theory [5]. Thus, $d$ and the jump of $v_{2}^{0}$ through $y=0$ are given by (12)-(15). In (12), $G$ represents the potential energy release rate corresponding to a non-cohesive crack with length $\ell$. It is related to the stress intensity factor $K_{I}$ of $\mathbf{u}$ by Irwin's formula. It remains to determine $\dot{\ell}$. From the optimal condition $\delta^{0}(-d)=\delta_{c}$, we obtain by induction and by virtue of (5) that $\delta_{c}=\sum_{i=1}^{\infty} \llbracket v_{2}^{0} \rrbracket(i \dot{\ell})$. Using (14), we obtain Eq. (16) giving $\dot{\ell}$ in terms of $G$. This equation has no solution if $G>G_{c}$, an infinite number of solutions if $G=G_{c}$ and a unique solution if $0<G<G_{c}$, see (17). Moreover, when $G / G_{c}$ is small, the relation between $\dot{\ell}$ and $G$ is approximately a power law with the power 2. In other words, we have obtained a propagation law which contains both Griffith and Paris laws: Griffith's law in the supercritical case where $G \geqslant G_{c}$ and Paris' law in the subcritical case where $G<G_{c}$. These results can be extended to the case of a general surface energy of Barenblatt type. Indeed, considering $\phi_{B}(\delta)$ given by (19), we show that $\mathrm{d} \ell / \mathrm{d} N$ is still proportional to $K_{I}^{4}$ for small $K_{I}$, cf. (26) where $C_{\phi}$ is given by (25) and $\bar{\delta}$ is solution of the integro-differential equation (24).

\section{Le modèle de forces cohésives et la démarche asymptotique}

On considère une structure bidimensionnelle $\Omega$ soumise à un chargement de déformation plane dépendant du temps provoquant la propagation d'une fissure suivant un trajet $\hat{\Gamma}$ connu a priori qui est une courbe régulière. Le matériau constitutif est linéairement élastique de coefficients de Lamé $\lambda$ et $\mu$ ( $E$ et $v$ étant le module d'Young et le coefficient de Poisson). On suppose que le chargement et le trajet de la fissure sont tels que la fissure sera toujours en mode I, i.e. que seule la composante normale du saut de déplacement $\llbracket \mathbf{u} \rrbracket \cdot \mathbf{n}$ est non nulle et que les cisaillements sont nuls sur $\hat{\Gamma}$. Pour définir la loi de forces cohésives sur $\hat{\Gamma}$, on prend l'ouverture cumulée comme variable $\delta$ mémorisant l'histoire de $\llbracket \mathbf{u} \rrbracket \cdot \mathbf{n}$ à travers $\hat{\Gamma}: \dot{\delta}=(\llbracket \dot{\mathbf{u}} \rrbracket \cdot \mathbf{n})^{+}$, le + désignant la partie positive et le point la dérivée temporelle. Envisageons tout d'abord comme densité surfacique d'énergie dissipée sur $\hat{\Gamma}$ celle correspondant au modèle de Dugdale, i.e. la fonction $\phi_{D}(\delta)=G_{c} \min \left\{\delta / \delta_{c}, 1\right\}$ où $\sigma_{c}$ est la contrainte de traction critique et $G_{c}$ est le taux de restitution d'énergie critique. Le rapport $\delta_{c}:=G_{c} / \sigma_{c}$ est l'ouverture critique, c'est une longueur caractéristique du matériau. 
Pour déterminer l'évolution de la fissuration, il suffit de déterminer le champ de déplacement $\mathbf{u}^{t}$ à chaque instant $t$ dans $\Omega$, son saut et l'ouverture cumulée $\delta^{t}$ sur $\hat{\Gamma}$ s'en déduisant ainsi que les contraintes $\sigma^{t}$ dans $\Omega$. On adopte l'approche variationnelle (quasistatique) développée dans [1] qui, après discrétisation en temps, se ramène à une séquence de problèmes de minimisation de l'énergie totale. C'est à partir de ce problème de minimisation incrémental (adapté au problème de décollement de film mince) que sont établies dans [2] et [3] toutes les propriétés de la loi de fatigue. Comme il est impossible de procéder de façon aussi déductive ici, nous faisons quelques hypothèses sur la structure de la solution.

On paramétrise $\hat{\Gamma}$ par son abscisse curviligne $s \in[0, L]$ et on l'identifie avec le segment $[0, L]$. A chaque instant $t$, on suppose que l'on peut distinguer trois parties dans $\hat{\Gamma}$ : (i) une zone $\Gamma_{t}^{d}=\left[0, \ell_{t}\right]$, dite zone non cohésive, où $\delta^{t} \geqslant \delta_{c}$; (ii) une zone $\Gamma_{t}^{c}=\left(\ell_{t}, \ell_{t}+d_{t}\right)$, dite zone cohésive, où $0<\delta^{t}<\delta_{c}$; (iii) une zone $\Gamma_{t}^{b}=\left[\ell_{t}+d_{t}, L\right]$, dite zone saine, où $\delta^{t}=0$, i.e. où aucun déplacement relatif n'a encore eu lieu. Ce faisant, $\ell_{t}$ et $d_{t}$ représentent respectivement la position de la pointe non cohésive et la longueur de la zone cohésive. Du fait de l'irréversibilité, $\ell_{t}$ et $\ell_{t}+d_{t}$ croissent avec $t$. Sur $\Gamma_{t}^{c}$, on distingue les zones $\Gamma_{t}^{c+}=\left\{s \in \Gamma_{t}^{c}: \llbracket \dot{\mathbf{u}}^{t} \rrbracket \cdot \mathbf{n}>0\right\}$ où l'ouverture augmente, $\Gamma_{t}^{c-}=\left\{s \in \Gamma_{t}^{c}: \llbracket \dot{\mathbf{u}}^{t} \rrbracket \cdot \mathbf{n}<0\right\}$ où l'ouverture diminue et la zone $\Gamma_{t}^{c 0}=\left\{s \in \Gamma_{t}^{c}\right.$ : $\left.\llbracket \dot{\mathbf{u}}^{t} \rrbracket \cdot \mathbf{n}=0\right\}$ où l'ouverture ne varie pas. On obtient alors

$$
\sigma^{t} \mathbf{n} \cdot \mathbf{n}=\sigma_{c} \quad \operatorname{sur} \Gamma_{t}^{c+}, \quad \sigma^{t} \mathbf{n} \cdot \mathbf{n}=0 \quad \operatorname{sur} \Gamma_{t}^{c-}, \quad \sigma^{t} \mathbf{n} \cdot \mathbf{n} \in\left[0,+\sigma_{c}\right] \quad \operatorname{sur} \Gamma_{t}^{c 0}
$$

Le champ $u^{t}$ ne peut être singulier qu'à la pointe $\ell_{t}+d_{t}$. La singularité, si elle existe, est une singularité classique en $K^{t} \sqrt{r} U(\theta)$ avec $(r, \theta)$ les coordonnées polaires de pôle la pointe de la zone cohésive et le déplacement $U(\theta)$ est donné dans (4). Les évolutions de $\ell_{t}$ et de $d_{t}$ sont obtenues en écrivant que l'ouverture cumulée à la pointe non cohésive est égale à l'ouverture critique et que $u^{t}$ ne doit pas être singulier à la pointe de la zone cohésive. En résumé, les deux équations donnant $\ell_{t}$ et $d_{t}$ sont

$$
\delta^{t}\left(\ell_{t}\right)=\delta_{c}, \quad K^{t}=0
$$

Supposons que la structure ait des dimensions grandes vis à vis de la longueur caractéristique du matériau, i.e. $\delta_{c} / L \ll 1$, et qu'elle soit soumise à un chargement cyclique "simple" dont l'intensité oscille entre 0 et $D$. Dans le cas du décollement d'un film mince [3], on en déduisait les trois propriétés importantes suivantes :

(i) L'avancée de la pointe non cohésive à chaque cycle est petite devant les dimensions de la structure ;

(ii) La longueur de la zone cohésive est petite devant les dimensions de la structure ;

(iii) D’un cycle au suivant, les champs varient peu, un régime quasi-stationnaire s'installe.

Nous les adoptons ici comme hypothèses de travail et vérifions leur cohérence a posteriori. Elles permettent de faire une séparation d'échelles, aussi bien en espace qu'en temps.

Soit $\ell$ de l'ordre de $L$ et donc grand devant $\delta_{c}$. De (i) on déduit qu'il faut un nombre de cycles $N$ grand (d'autant plus grand que $\delta_{c} / L$ est petit) pour que la pointe non cohésive arrive en $\ell$. Envisageons les cycles voisins de $N$, i.e. les cycles $N+i$ avec $i \in\{0, \pm 1, \pm 2, \ldots\}$. On peut distinguer deux échelles de nombre de cycles : la grande échelle $N$ et la petite échelle $i$. Intéressons-nous aux variations de la fissure à petite échelle autour de $N$. Le paramètre temporel $t$ est choisi de façon à ce que $t=i$ à la fin de la montée en charge du cycle $i$. (L'intervalle de temps du cycle $i$ est donc $(i-1, i]$.

De (i) et (iii) on déduit que l'avancée de la fissure à chaque cycle autour de $N$ est petite et indépendante de $i$. Notons-la $\dot{\ell}$. Grâce à son indépendance par rapport à $i, \dot{\ell}$ s'identifie avec le taux d'avancée à grande échelle de la fissure $\mathrm{d} \ell / \mathrm{d} N$. De plus, on déduit de (ii) que la longueur de la zone cohésive à la fin de chaque cycle autour de $N$ est petite et indépendante de $i$. Notons-la $d$. Par conséquent le déplacement $\mathbf{u}^{i}$ à la fin de la montée en charge du cycle $i$ est en première approximation égal à celui correspondant à une fissure de longueur $\ell$ sans zone cohésive, notons ce dernier $\mathbf{u}$ (qui dépend donc de $N$ mais pas de $i$ ). De façon précise, u vérifie le problème à grande échelle suivant (en plus de la donnée du chargement $D$ ) :

$$
\operatorname{div} \sigma=0, \quad \sigma=\lambda \operatorname{div} \mathbf{u} I+2 \mu \varepsilon(\mathbf{u}), \quad \operatorname{dans} \Omega \backslash \hat{\Gamma}, \quad \sigma \mathbf{n}=0 \quad \text { sur }(0, \ell), \quad \llbracket \mathbf{u} \rrbracket=0 \quad \text { sur }[\ell, L]
$$

Ce champ u est singulier à la pointe $\ell$ avec une singularité en $\sqrt{r}$ et un facteur d'intensité des contraintes $K_{I}$ (qui dépend de $\ell$ et est proportionnel à $D$ ) :

$$
\mathbf{u}(\mathbf{x})=\frac{K_{I}}{2 \mu} \sqrt{\frac{r}{2 \pi}} U(\theta)+\text { partie régulière, } \quad U(\theta)=(3-4 v-\cos \theta)\left(\cos \frac{\theta}{2} \mathbf{e}_{1}+\sin \frac{\theta}{2} \mathbf{e}_{2}\right)
$$


où $(r, \theta)$ sont les coordonnées polaires avec comme origine la pointe de la fissure et comme $\theta=0$ la direction de la tangente à $\hat{\Gamma}$ en $\ell$. Toutefois, $\mathbf{u}$ ne sert d'approximation de $\mathbf{u}^{i}$ qu'assez loin de la zone cohésive. Or on a besoin d'avoir une bonne approximation de $\mathbf{u}^{i}$ au voisinage de la zone cohésive pour déterminer $\dot{\ell}$. Cela passe par la résolution d'un problème dit à petite échelle que nous présentons ci-dessous.

\section{Le problème local et la loi de fatigue dans le cas du modèle de Dugdale}

On prend comme origine du repère la pointe $\ell+d$ de la zone cohésive au cycle $N$, comme axe 1 la tangente à $\hat{\Gamma}$ en $\ell+d$ orientée dans le sens des $s$ croissants et on fait un changement d'échelle d'espace à l'aide d'un zoom. Le domaine à petite échelle est alors le plan $\mathbb{R}^{2}$ muni du système de coordonnées $(x, y)$. On note $\mathbf{v}^{i}$ le champ de déplacement régnant dans ce domaine à la fin de la montée en charge du cycle $i$ qui servira d'approximation locale de $\mathbf{u}^{i}$. Du fait de l'existence supposée d'un régime stationnaire à petite échelle de cycles (cf. la propriété (iii)), tous les $\mathbf{v}^{i}$ se déduisent de $\mathbf{v}^{0}$ par translation :

$$
\mathbf{v}^{i}(x, y)=\mathbf{v}^{0}(x-i \dot{\ell}, y), \quad i \in \mathbb{Z}
$$

En $i=0$ la zone non cohésive $\gamma_{0}^{d}$ est le demi-axe $(-\infty,-d] \times\{0\}$ et la zone cohésive $\gamma_{0}^{c}$ le segment $(-d, 0) \times\{0\}$. Le chargement à l'infini est celui de la singularité de $\mathbf{u}$ (avec $K_{I}$ pour facteur d'intensité des contraintes).

Il reste à déterminer $\mathbf{v}^{0}, d$ et $\dot{\ell}$. On suppose a priori que la zone cohésive est entièrement active, i.e. $\gamma_{0}^{c}=\gamma_{0}^{c+}$, et on vérifie $a$ posteriori la cohérence de cette hypothèse. (Cette vérification passe par le calcul du champ de déplacement $\mathbf{v}^{t}$ à chaque instant $t \in(-1,0]$ du cycle 0 .) Le champ $\mathbf{v}^{0}$ est donc tel que

$$
\begin{aligned}
& \operatorname{div} \sigma^{0}=0, \quad \sigma^{0}=\lambda \operatorname{div} \mathbf{v}^{0} I+2 \mu \varepsilon\left(\mathbf{v}^{0}\right) \quad \operatorname{dans} \mathbb{R}^{2} \backslash\left(\gamma_{0}^{d} \cup \gamma_{0}^{c}\right) \\
& \sigma^{0} \mathbf{e}_{2}=0 \quad \text { sur } \gamma_{0}^{d}, \quad \sigma^{0} \mathbf{e}_{2}=\sigma_{c} \mathbf{e}_{2} \quad \text { sur } \gamma_{0}^{c}
\end{aligned}
$$

avec la condition à l'infini

$$
\lim _{r \rightarrow \infty}\left(\mathbf{v}^{0}(x, y)-\frac{K_{I}}{2 \mu} \sqrt{\frac{r}{2 \pi}} U(\theta)\right)=0 \quad \text { où } x=r \cos \theta, y=r \sin \theta
$$

À $d$ donné, ce problème admet une solution $\mathbf{v}^{0}$ unique qui s'obtient explicitement à partir de la méthode des potentiels complexes, cf. [5]. On ne donne ici que les principaux résultats. Les champs $\mathbf{v}^{0}$ et $\sigma^{0}$ se déduisent de la fonction $\varphi(z)$ de la variable complexe $z=x+\mathrm{i} y, \mathrm{i}=\sqrt{-1}$, grâce aux relations

$$
\begin{aligned}
& \sigma_{22}^{0}(x, y)-\mathrm{i} \sigma_{12}^{0}(x, y)=\varphi^{\prime}(z)+\varphi^{\prime}(\bar{z})+(z-\bar{z}) \overline{\varphi^{\prime \prime}(z)} \\
& 2 \mu\left(v_{1}^{0}(x, y)+\mathrm{i} v_{2}^{0}(x, y)\right)=(3-4 v) \varphi(z)-\varphi(\bar{z})-(z-\bar{z}) \overline{\varphi^{\prime}(z)}
\end{aligned}
$$

$\varphi$ étant holomorphe dans le plan complexe privé de la demi-droite $\gamma_{0}^{d} \cup \gamma_{0}^{c}$, la barre désignant le complexe conjugué. À partir de la répartition de forces cohésives donnée par (7) et de la condition à l'infini (8), on trouve

$$
\varphi^{\prime}(z)=\frac{\sigma_{c}}{2 \pi \sqrt{z}} \int_{-d}^{0} \frac{\sqrt{-x}}{x-z} \mathrm{~d} x+\frac{K_{I}}{2 \sqrt{2 \pi z}}
$$

La longueur de la zone cohésive $d$ s'obtient en écrivant qu'il n'y a pas de singularité à la pointe cohésive, cf. (2) 2 . D'où

$$
\begin{aligned}
& d=\frac{\pi}{8\left(1-v^{2}\right)} \frac{E}{\sigma_{c}} \frac{G}{G_{c}} \delta_{c} \quad \text { avec } G=\frac{1-v^{2}}{E} K_{I}^{2} \\
& \varphi^{\prime}(z)=\frac{\sigma_{c}}{2}+\frac{\mathrm{i} \sigma_{c}}{2 \pi}(\log (\sqrt{d}+\mathrm{i} \sqrt{z})-\log (\sqrt{d}-\mathrm{i} \sqrt{z}))
\end{aligned}
$$

Dans (12), $G$ représente le taux de restitution d'énergie potentielle associé à la structure comportant une fissure non cohésive de longueur $\ell$, cf. le problème à grande échelle (3). Il est relié à $K_{I}$ par la formule d'Irwin. Dans (13), Log désigne la détermination principale du logarithme complexe. On en déduit que le saut du déplacement normal est donné par

$$
\llbracket v_{2}^{0} \rrbracket(x)=V\left(1+\frac{x}{d}\right) \frac{G}{G_{c}} \delta_{c}, \quad x \geqslant-d
$$


où $V$ désigne la fonction définie sur $\mathbb{R}_{*}^{+}$par

$$
V(l)= \begin{cases}\sqrt{1-l}+l \ln (1-\sqrt{1-l})-l \ln \sqrt{l} & \text { si } 0<l \leqslant 1 \\ 0 & \text { si } l \geqslant 1\end{cases}
$$

et prolongée par continuité par 1 en 0 . (La valeur du saut pour $x<-d$ n'est pas donnée ici, étant inutile pour la suite.)

Pour obtenir $\dot{\ell}$ on écrit, conformément à $(2)_{1}, \delta^{0}(-d)=\delta_{c}$. À partir du calcul de $\mathbf{v}^{t}$ pour $t \in(-1,0]$, on montre que $\llbracket \dot{v}_{2}^{t} \rrbracket \leqslant 0$ durant la décharge (i.e. quand $-1<t<-1 / 2$ ), que $\mathbf{v}^{-1 / 2}=0$ (i.e. les déplacements sont nuls à la fin de la décharge) et que $\llbracket \dot{v}_{2}^{t} \rrbracket \geqslant 0$ durant la charge (i.e. quand $\left.-1 / 2<t<0\right)$. Ceci permet d'écrire $\delta^{0}=\delta^{-1}+\llbracket v_{2}^{0} \rrbracket$. Grâce à (5) et en procédant par induction, on obtient $\delta^{0}(x)=\sum_{i=0}^{\infty} \llbracket v_{2}^{0} \rrbracket(x+i \dot{\ell})$ qui, grâce à (12) et (14), fournit l'équation cherchée en $\dot{\ell}$ :

$$
1=\frac{G}{G_{c}} \sum_{i=0}^{\infty} V\left(\frac{i \dot{\ell}}{d}\right)
$$

Cette équation diffère légèrement de celle obtenue dans [4] pour une fissure en mode III, mais possède les mêmes propriétés, à savoir

(i) Si $G>G_{c}$, alors il n'y a pas de solution;

(ii) Si $G=G_{c}$, alors tout $\dot{\ell} \geqslant \frac{\pi}{8\left(1-v^{2}\right)} \frac{E}{\sigma_{c}} \delta_{c}$ est solution;

(iii) Si $0<G<G_{c}$, alors il existe une seule solution qui peut s'écrire

$$
\dot{\ell}=\frac{\pi}{8\left(1-v^{2}\right)} \frac{E}{\sigma_{c}} \mathrm{f}\left(\frac{G}{G_{c}}\right) \delta_{c}
$$

$g \mapsto \mathrm{f}(g)$ étant une fonction (sans dimension) de classe $C^{1}$, strictement croissante de 0 à 1 quand $g$ croît de 0 à 1 . (iv) Pour des faibles valeurs de $g=G / G_{c}$, on a $\mathrm{f}(g)=g^{2} / 3+o\left(g^{2}\right)$ et on obtient la loi puissance

$$
\dot{\ell} \approx \frac{\pi}{24\left(1-v^{2}\right)} \frac{E}{\sigma_{c}}\left(\frac{G}{G_{c}}\right)^{2} \delta_{c}
$$

Autrement dit, la loi de fatigue obtenue contient la loi de Griffith puisqu'elle interdit des valeurs de $G>G_{c}$ et qu'elle laisse indéterminée $\dot{\ell}$ quand $G=G_{c}$. En régime sous-critique, i.e. quand $G<G_{c}$, elle constitue une loi de type Paris, le taux d'avancée de la fissure étant fonction du taux de restitution d'énergie. Elle s'apparente à une loi puissance en $G^{2}$ et donc en $K^{4}$ pour des faibles valeurs de $G / G_{c}$.

\section{Cas des modèles de forces cohésives plus généraux}

Envisageons maintenant des modèles de forces cohésives plus généraux. Conservons l'ouverture cumulée comme variable mémoratrice et prenons pour densité d'énergie de surface une fonction $\phi_{B}$ qui contient toujours les deux paramètres matériaux $G_{c}$ et $\delta_{c}$ et qui peut s'écrire (le prime désignant la dérivée)

$$
\phi_{B}(\delta)=G_{c} \phi\left(\delta / \delta_{c}\right), \quad \phi(0)=0, \quad \phi(+\infty)=1, \quad \phi^{\prime} \geqslant 0, \quad \phi^{\prime \prime} \leqslant 0
$$

où $\phi$ est une fonction sans dimension, définie sur $[0,+\infty)$, continûment différentiable, concave, nulle en 0 , croissante et tendant vers 1 à l'infini. Si l'on reprend le problème à petite échelle, seules les conditions aux limites sur les lèvres de la fissure changent, (7) devenant

$$
\sigma^{0} \mathbf{e}_{2}=\sigma_{c} \phi^{\prime}\left(\delta^{0} / \delta_{c}\right) \mathbf{e}_{2} \quad \text { sur } \gamma_{0}=(-\infty, 0) \times\{0\}
$$

où $\sigma_{c}=G_{c} / \delta_{c}$. Il n'y a plus lieu d'introduire une zone non cohésive comme dans le modèle de Dugdale, car les forces cohésives peuvent ne jamais s'annuler (c'est le cas si $\phi^{\prime}>0$ ). Ceci n'est pas contradictoire avec l'hypothèse de travail (ii), ce n'est qu'une question d'échelle. Les forces cohésives sont finies à une échelle de longueur donnée $\operatorname{par} \delta_{c}$ et sont négligeables à grande échelle. Autrement dit, maintenant $d$ est $a$ priori infini à petite échelle et il reste à trouver $\mathbf{v}^{0}, \delta^{0}$ et $\dot{\ell}$. En supposant connu $\delta^{0}, \mathbf{v}^{0}$ s'obtient à partir du potentiel complexe $\varphi$ qui maintenant est donné par

$$
\varphi^{\prime}(z)=\frac{\sigma_{c}}{2 \pi \sqrt{z}} \int_{-\infty}^{0} \phi^{\prime}\left(\frac{\delta^{0}(x)}{\delta_{c}}\right) \frac{\sqrt{-x}}{x-z} \mathrm{~d} x+\frac{K_{I}}{2 \sqrt{2 \pi z}}
$$


En retour et en supposant que $\mathbf{v}^{t}$ décroît jusqu'à $\mathbf{0}$ lors des décharges et crôit lors des charges, $\delta^{0}$ reste lié à $\mathbf{v}^{0}$ et $\dot{\ell}$ par la relation $\delta^{0}(x)=\sum_{i=0}^{+\infty} \llbracket v_{2}^{0} \rrbracket(x+i \dot{\ell})$. Enfin, $\dot{\ell}$ est obtenu en écrivant qu'il n'y a pas de singularité à la pointe de la fissure $z=0$. En résumé, $\mathbf{v}^{0}, \delta^{0}$ et $\dot{\ell}$ sont donnés par le système intégro-différentiel non linéaire suivant :

$$
\begin{aligned}
& \frac{\pi E}{4\left(1-v^{2}\right) \sigma_{c}} \llbracket v_{2}^{0} \rrbracket^{\prime}(x)=\int_{-\infty}^{0} \phi^{\prime}\left(\frac{\delta^{0}(s)}{\delta_{c}}\right) \frac{\sqrt{-x} \mathrm{~d} s}{(x-s) \sqrt{-s}} \\
& \delta^{0}(x)=\sum_{i=0}^{+\infty} \llbracket v_{2}^{0} \rrbracket(x+i \dot{\ell}), \quad \frac{K_{I}}{\sqrt{2 \pi}}=\frac{\sigma_{c}}{\pi} \int_{-\infty}^{0} \phi^{\prime}\left(\frac{\delta^{0}(s)}{\delta_{c}}\right) \frac{\mathrm{d} s}{\sqrt{-s}}
\end{aligned}
$$

l'intégrale dans (22) étant prise au sens de la valeur principale en raison de la présence du noyau intégral. L'étude tant théorique que numérique de ce système sort du cadre de cette Note. On suppose qu'une solution existe et on se contente d'en extraire quelques propriétés lorsque $K_{I}$ est petit en s'appuyant sur des arguments heuristiques.

Lorsque $K_{I}$ est petit (i.e. quand $G / G_{c}$ est petit devant 1 ), $\dot{\ell}$ est petit et la relation (23) 1 peut se simplifier. En effet, comme $\llbracket v_{2}^{0} \rrbracket(x)=0$ si $x \geqslant 0$, la somme est finie. Pour $x<0$ donné, $i$ varie de 0 à $n(x)=-x / \dot{\ell}$. On peut donc écrire $\dot{\ell} \delta^{0}(x)=-x \sum_{i=0}^{n(x)} \frac{1}{n(x)} \llbracket v_{2}^{0} \rrbracket(x(1-i / n(x)))$. Comme $\dot{\ell}$ est petit, $n(x)$ est grand et la somme peut être approchée par une intégrale, soit $\dot{\ell} \delta^{0}(x)=-x \int_{0}^{1} \llbracket v_{2}^{0} \rrbracket((1-\theta) x) \mathrm{d} \theta$, dont on déduit facilement $\llbracket v_{2}^{0} \rrbracket(x)=-\dot{\ell} \delta^{0^{\prime}}(x)$. Ceci fournit un procédé de construction de la solution de (22)-(23). On procède comme suit après introduction de grandeurs sans dimension (surmontées d'une barre),

$$
\bar{x}=\frac{\sigma_{c}^{2} x}{\sqrt{C_{\phi}} K_{I}^{2}}, \quad \bar{s}=\frac{\sigma_{c}^{2} s}{\sqrt{C_{\phi}} K_{I}^{2}}, \quad \bar{\delta}(\bar{x})=\frac{\delta^{0}(x)}{\delta_{c}}
$$

où $C_{\phi}$ est une constante sans dimension à déterminer. On résout tout d'abord l'équation intégro-différentielle en $\bar{\delta}$ suivante (qui ne fait intervenir que la fonction $\phi$ et où l'intégrale est prise au sens de la valeur principale)

$$
\frac{\mathrm{d}^{2} \bar{\delta}}{\mathrm{d} \bar{x}^{2}}(\bar{x})=\sqrt{-\bar{x}} \int_{-\infty}^{0} \frac{\phi^{\prime}(\bar{\delta}(\bar{s}))}{\bar{s}-\bar{x}} \frac{\mathrm{d} \bar{s}}{\sqrt{-\bar{s}}}, \quad \bar{x}<0, \quad \bar{\delta}(0)=\frac{\mathrm{d} \bar{\delta}}{\mathrm{d} \bar{x}}(0)=0
$$

On pose ensuite

$$
C_{\phi}=\frac{\pi^{2}}{4}\left(\int_{-\infty}^{0} \phi^{\prime}(\bar{\delta}(\bar{s})) \frac{\mathrm{d} \bar{s}}{\sqrt{-\bar{s}}}\right)^{-4}
$$

et on vérifie enfin que $\delta^{0}, \llbracket v_{2}^{0} \rrbracket$ et $\dot{\ell}$ donnés par

$$
\delta^{0}(x)=\bar{\delta}(\bar{x}) \delta_{c}, \quad \llbracket v_{2}^{0} \rrbracket(x)=-\dot{\ell} \frac{\mathrm{d} \delta^{0}}{\mathrm{~d} x}(x), \quad \dot{\ell}=\frac{4 C_{\phi}}{\pi\left(1-v^{2}\right)} \frac{E}{\sigma_{c}}\left(\frac{G}{G_{c}}\right)^{2} \delta_{c}
$$

sont solutions de (22)-(23) (compte-tenu de l'approximation de la série par une intégrale). On voit que la constante $C_{\phi}$ ne dépend que de la fonction énergie de surface $\phi$ et que la loi de fatigue reste une loi de Paris avec un exposant 4 en terme du facteur d'intensité des contraintes (pour des faibles valeurs de celui-ci). Seule la constante $C_{\phi}$ change d'un modèle à l'autre et nécessite la résolution (en général numérique) de (24). En appliquant cette méthode au cas de l'énergie de surface de Dugdale, i.e. $\phi(\bar{\delta})=\min \{\bar{\delta}, 1\}$, on retrouve évidemment les résultats de la section précédente, la constante $C_{\phi}$ valant $\pi^{2} / 96$.

\section{Conclusion et perspectives}

Cette étude complète celle faite dans [4] dans le cas d'une fissure en mode III et en renforce les résultats. En partant d'un modèle de force cohésive de type Dugdale-Barenblatt, on peut construire explicitement une loi de fatigue de type Paris, i.e. $\mathrm{d} \ell / \mathrm{d} N=f(K)$. De plus, pour des faibles valeurs du facteur d'intensité des contraintes, celle loi est une loi en $C K^{4}$ aussi bien en mode I qu'en mode III, que l'on prenne pour variable mémoratrice le glissement cumulé ou 
l'ouverture cumulée, que l'on prenne le modèle de Dugdale ou un modèle plus général de Barenblatt. Pour trouver des exposants différents on pourrait envisager d'autres comportements volumiques. Il serait également intéressant d'étudier l'influence de la forme du cycle de chargement sur la loi de fatigue. La généralisation au 3D est aussi un objectif à terme.

\section{Références}

[1] B. Bourdin, G.A. Francfort, J.-J. Marigo, The variational approach to fracture, J. Elasticity 91 (1-3) (2008) 5-148.

[2] A. Jaubert, J.-J. Marigo, L'approche variationnelle de la fatigue : des premiers résultats, C. R. Mecanique 333 (7) (2005) $550-556$.

[3] A. Jaubert, J.-J. Marigo, Justification of Paris-type fatigue laws from cohesive forces model via a variational approach, Contin. Mech. Thermodyn. 18 (1) (2006) 23-45.

[4] R. Abdelmoula, J.-J. Marigo, T. Weller, Construction d'une loi de fatigue à partir d'un modèle de forces cohésives : cas d'une fissure en mode III, C. R. Mecanique 337 (1) (2009) 53-59.

[5] N.I. Mushkelishvili, Some Basic Problems in the Mathematical Theory of Elasticity, Noordhoff, 1953. 